\title{
An Advance Approach of Face Recognition using PCA and Region Base Color Segmentation
}

\author{
Santosh Kumar \\ M.tech. IT \\ Gov. Engineering College, Ajmer
}

\author{
Manish Mathuria \\ M.tech. IT \\ Gov. Engineering College, Ajmer
}

\author{
Atul Chaudhary \\ Astt. Professor Dept. of IT \\ Gov. Engineering College, Ajmer \\ Kailash Rathore \\ M.tech. IT \\ Gov. Engineering College, Ajmer
}

\begin{abstract}
ABSTARCT
Automated face recognition has industrialized a major field of Interest Face Recognition is the typical process of identification of a individual by their facial image. This effective technique Making it possible to use the facial images of a person to authenticate him into a protected system, for criminal identification, for passport verification, entrance control in buildings, access control at automatic teller machines the experimentation involved Eigen faces and PCA (Principal Component Analysis). Recognition rate of $90 \%$ was achieved using combination of PCA and region base colour segmentation face recognition techniques.
\end{abstract}

Keywords: Face Recognition, PCA (Principal Component Analysis), and segmentation

\section{INTRODUCTION}

The face is our major focus of consideration social interaction, playing a major role to express identity and emotion. We can recognize many of faces lettered through our lifetime and identify familiar faces at a glint after years of interval. This skill is quite robust, disparagement large changes in the visual input due to viewing conditions, expression, aging, and misdirection such as sceptical or changes in hairstyle or facial expression. Computation models of face recognition, in exact, are remarkable because they can provide not only to theoretical inspiration but also to pragmatic applications. Computers that recognize faces can be applied to a wide variety of problems, including criminal identification, security systems, image and film processing, and human computer interaction. Unfortunately, developing a computation model of face recognition is some time very difficult, because faces are very complex, multidimensional, and meaningful visual motivations. The user should focus his attention toward developing a sort of early, pre attentive Pattern recognition capability that does not depend on having three-dimensional information or detailed geometry. He should develop a computation model of face recognition that is fast, reasonably simple, and exact. Self-learning capabilities and later recognizing new faces is pragmatic most within this framework. Face Recognition under different broadly varying conditions is completed by training on a limited number of representative display The method have some advantages over other face recognition pattern in its speed and step by step simplicity learning capability .[1]

Images of faces, represented as high-dimensional pixel arrays, habitually belong to a multiple of as a such low dimension. Face recognition, and computer idea research in general, has watching a growing awareness in techniques that exploit on this performance, and apply algebraic and statistical tools for extraction and analysis of the principal multiple. Eigen face is a part of feature extraction face recognition technique that can match or compare subject face.

\section{RELATED WORK}

The identification of any person maybe found many of different techniques those techniques have some advantage and some have dis advantage, which must be considered in biometrical system development like system price, reliability, robustness, necessity of physical contact with scanning device and many others things. face is detect and recognise. First face is capture by camera and after that some process which may be any method of feature extraction after those process extracted features are stored. Same process is also occur in other side extracted feature is matched in comparing block [5.1]

\subsection{Fingerprints}

Fingerprints is always unique for each finger of a person comprises identical twins. One of the most commercially manner available biometric technologies, fingerprint recognition devices for desktop and laptop access are now usually available from many different laptop makers at a low cost.[6]

\subsection{Iris Recognition}

This recognition method using the iris of the eye, which is the coloured area of eye that surrounds the pupil (The pupil is a hole located in the center of the iris of the eye that allows light to enter the retina) Iris patterns are thought unique. The iris patterns are get through a video-based image accomplish system. Iris scanning devices have been used in personal authentication applications for many years The technology works well in both verification and identification modes Current systems can be used even in the presence of eye glasses sunglass and contact lenses[7] 


\subsection{Signature Verification}

A digital signature is a mathematical schema for prove the authenticity of a digital message or document. A valid digital signature (A digital signature is a mathematical scheme for signifying the authenticity of a digital message or document) gives a recipient reason to believe that the message was created by a known sender, that send the message or we can say that digital signature authenticate the message sender identity Digital signatures are mostly used for software supply, commercial transactions, and in other cases where it is important to detect forgery or message interfering cases.

\subsection{Face Recognition}

Biometric-based techniques have developed as the most promising option for recognizing personages in recent years since, instead of authenticating people and allowing them access to physical and virtual domains based on passwords, PINs, smart cards, pliable identification cards, tokens, keys and so forth, these methods examine an individual's physiological and/or behavioural characteristics I order to determine and/or establish his individuality. Keywords and replicated magnetic cards can become corrupted and unreadable. However, an individual's biological behaviours Cannot be misplaced, forgotten, stolen or bogus.

\subsection{Voice Recognition}

Voice recognition is the special technology by which sounds, words or slogans spoken by persons are converted into electrical signals forms, and those signals forms are transformed into some coding patterns to which meaning has been assigned While the concept could more usually be socalled "sound recognition The main effort in using voice as an input to a computer simulation lies in the fundamental variances between human speech and the more outmoded forms of computer input.[6] Although computer programs are commonly designed to produce an accurate and well-defined reaction upon receiving the appropriate (and equally accurate) input, the human voice and spoken words are anything but accurate. Each human voice is different, and identical words can have different meanings if spoken with different dissimilarities or in different contexts. Several approaches have been tried, with varying degrees of success, to amaze these problems.

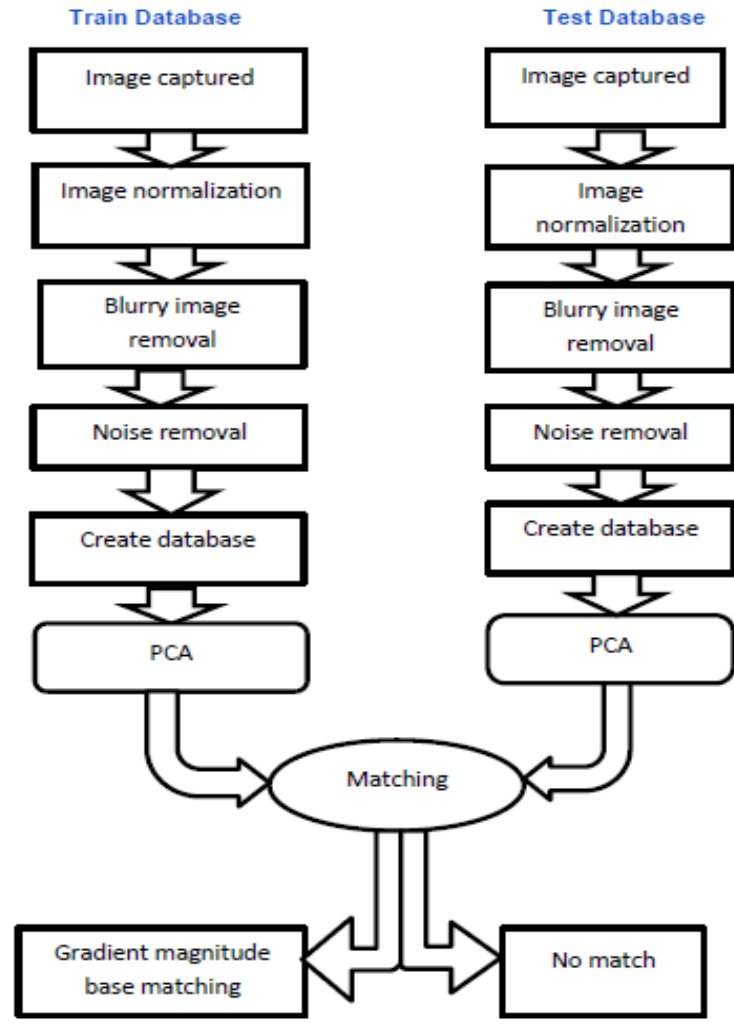

Fig 1: proposed methodology

\section{REGION BASE COLOR SEGMENTATION}

Region base color segmentation is mainly a logical Application for face tracking, face expression recognition etc. in image segmentation is process where assigning a label to each and every pixel Image segmentation means division of an image into meaningful structure. It is the process of extraction and representing information from the image to group pixels together with region of similarity. In the seed based color image segmentation method selection of initial seed is crucial step because it decides the overall segmentation by region growing technique. 


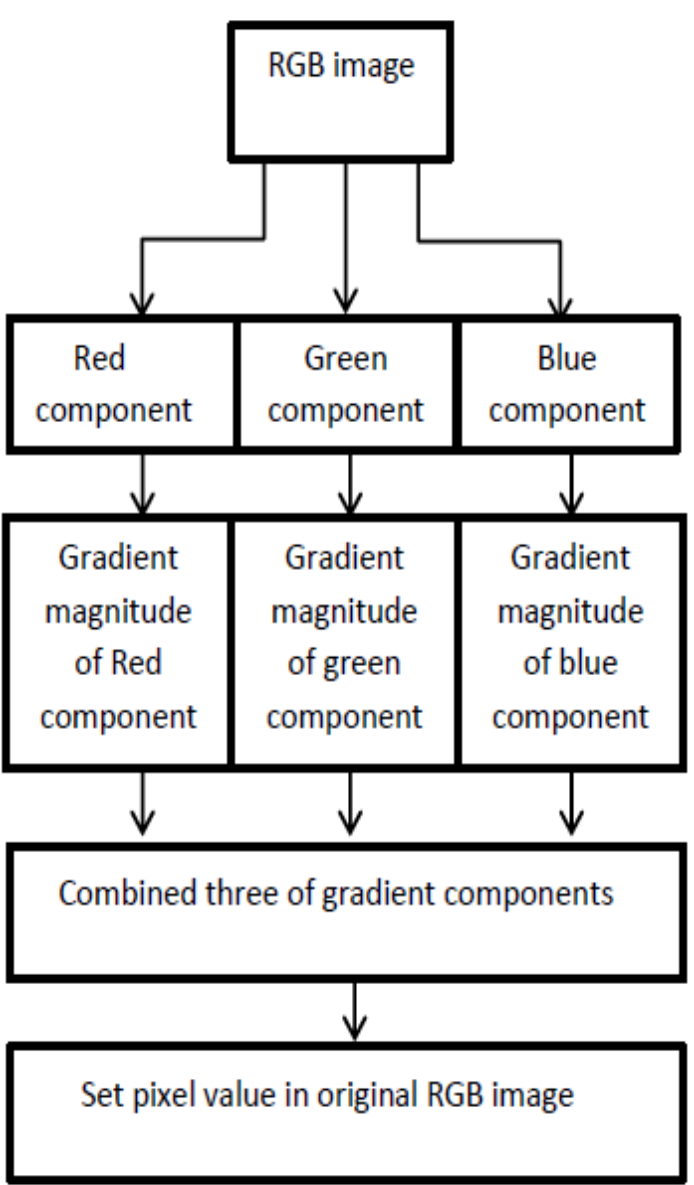

Fig 2 region base color segmentation

In fig 3 A block diagram is given above a RGB model is a basic color model of any color image red green blue color igg added together to produce many number of color. Magnitude of RGB color is calculated and combined it lastly set the pixel value in original RGB image

\section{PRINCIPAL COMPONENT}

\section{ANALYSIS}

PCA is a useful modern statistical technique that has used application in fields such as face recognition and image compression, and is a common technique for finding patterns in data of high dimension. PCA is a commonly used method of object recognition as its results, when used properly can be fairly accurate and resilient to noise. The method of which PCA is applied can vary at different stages so what will be demonstrated is a clear method for PCA application that can be followed. It is up for individuals to experiment in finding the best method for producing accurate results from PCA and PCA Need less time to recognise the face, PCA can do prediction, redundancy removal, feature extraction and data comparison fig

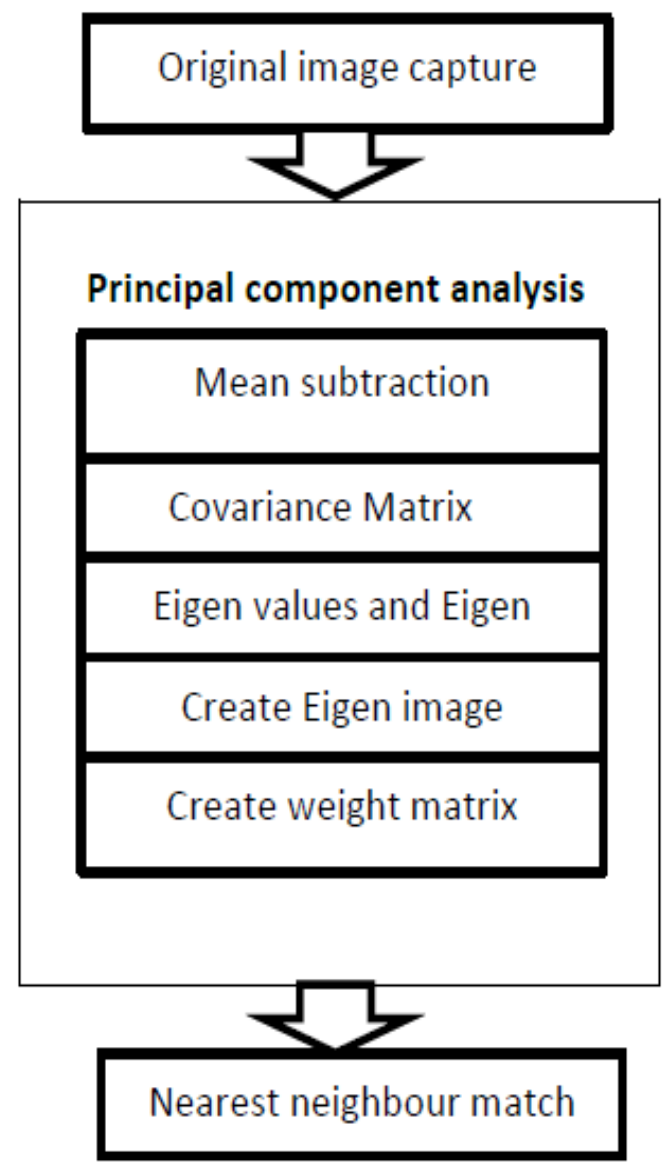

Fig 3 PCA based block diagram

\section{PRAPOSED METHODOLOGY}

Main methodology or workflow model is in this paper is shown in fig 1 A image is first captured by the static camera most of the time captured image may be blurry and have some noise both things produce problem so before create database we have to remove both things after that use Some steps of face recognition using PCA (Principle Component Analysis) if image is matched then apply region base colour segmentation is done.

\subsection{Step 1}

After getting image matrix in proposed system creates mean matrix the dimension of the mean matrix must to be square. Mean matrix calculated by adding total value of column and those will be divided by number of column.

Mean $=1 / N \sum_{k=1}^{n} I \mathrm{k}$

\subsection{Step 2}

Subtract mean from each from each image matrix which is taken previously after that we get the mean subtracted data

$$
\mathrm{A}=\left[\left(I_{1} \text { - mean }\right),\left(I_{2} \text {-mean }\right),\left(I_{n} \text {-mean }\right)\right]
$$

\subsection{Step 3}

Calculate the covariance matrix is the main step of principle component analysis.

$\mathrm{C}=\mathrm{A} A^{T}$ 
As $\operatorname{Dim}(\mathrm{A})=(\mathrm{m} * \mathrm{n}) \times \mathrm{N}$ and $\operatorname{Dim}\left(A^{T}\right)=\mathrm{N} \times(\mathrm{m} * \mathrm{n}) \mathrm{SO}$ $\operatorname{Dim}(C)=\left(m^{*} n\right) x\left(m^{*} n\right)$ which is very large and computation will be difficult. So another matrix has been created by rearranging

$\operatorname{Dim}(\mathrm{L})=\mathrm{N} \times \mathrm{N}$ which is easy to handle

\subsection{Step 4}

find Eigen value and Eigen vector from obtained image matrix. Then the system finds the Eigen vectors and Eigen values. For $\mathrm{N}$ dimensional vector there will be $\mathrm{N}$ Eigen values and Eigen vectors.

\subsection{Step 5}

Its main part of the principal component analysis create Eigen faces from train data base and and extract principal. The proposed system then creates an Eigen image by multiplying mean subtracted data matrix with the Eigen vectors.

\section{Eigimage $=(\mathrm{A})($ Eigvec $)$}

The main indication of principal component analysis is to discovery the vectors that best excuse for the distribution of face images within the whole image space. These vectors express the subspace of face images, which

we call "face space". Each vector is of length N square, describes an N-by-N image, and is a linear combination of original face images, and because they are face-like in appearance, we refer then to as "Eigen faces".

\subsection{Step 6}

The main data set or we called final data set is obtained which is used for identification or matching process weight matrix has been created by multiplying the transposed large Eigen image with the mean subtracted data matrix

Weight Mat $=(\operatorname{lrgEigenimageT})(\mathrm{A})$

When this step is completed we can say that image is ready to compare and matching using weight matrix.

\subsection{Step 7}

Now the train database image and test data base real image will be match after completion of the principal component analysis main steps(step1 to step6)

\subsection{Step 8}

After success full matching of text database image from train database image we can apply region base color segmentation on both matched images and get the gradient magnitude result. Using these steps we can achieve much more accuracy level.

\section{EXPERIMENTAL RESULT}

The proposed methodology experimental process is operate with LG 5 mega pixel static camera and picture is not click more than five to six feet captured image at a spatial resolution of $180 \times 200$ pixels frame rate is not more than 25 frame per second. A low rate camera is used for this experiment because our goal is also research design must be economical.
A simple example of Eigen faces is shown in figure below

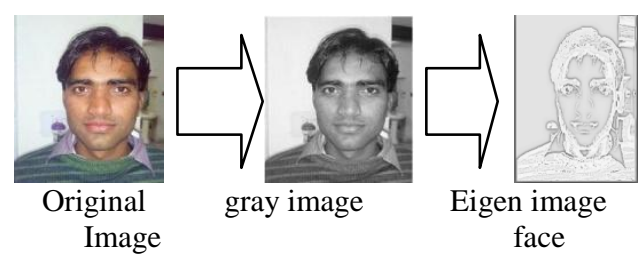

Fig 4: Eigen image conversion

Above fig. 4 shows that how the original captured image is converted to first gray image and after that using PCA algorithm its will be converted into Eigen faces. Those Eigen image face is extracted image which should be match with train database image.

Table 1: Data

\begin{tabular}{|l|l|l|}
\hline $\mathbf{1}$ & $\begin{array}{l}\text { Number of image trained } \\
\text { database }\end{array}$ & 10 \\
\hline $\mathbf{2}$ & Number of image in test database & 5 \\
\hline $\mathbf{3}$ & False positive(wrong detection) & 0 \\
\hline $\mathbf{4}$ & Time to execute & $0.683 \mathrm{sec}$ \\
\hline $\mathbf{5}$ & Accuracy level & $90 \%$ \\
\hline
\end{tabular}

The performance of the proposed face recognition system is tasted with different image poses of same person

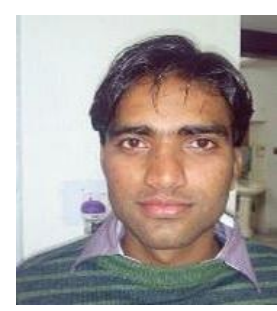

1

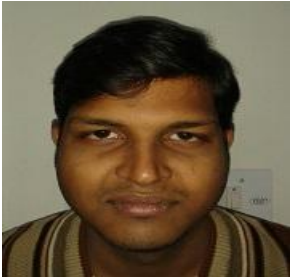

3

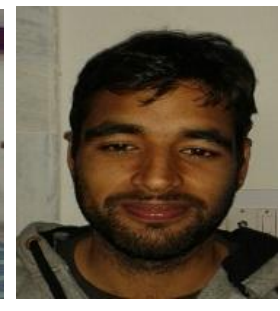

2

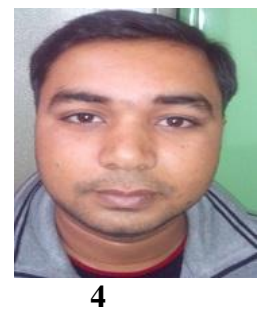

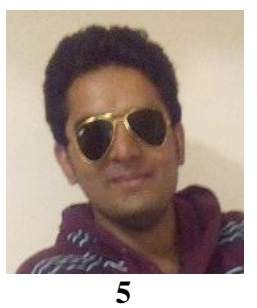

Fig.5: Test database 


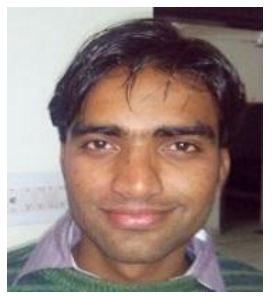

1

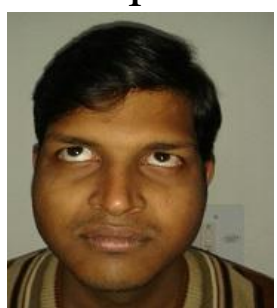

3

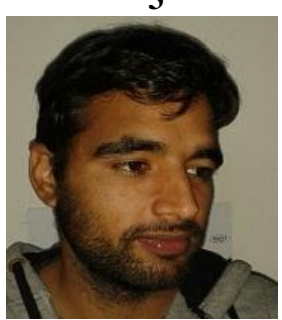

5

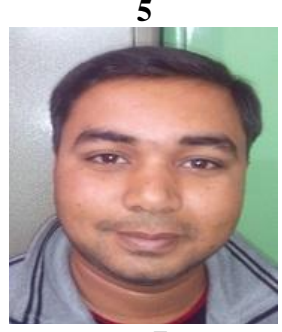

7

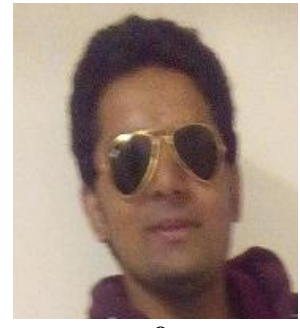

9

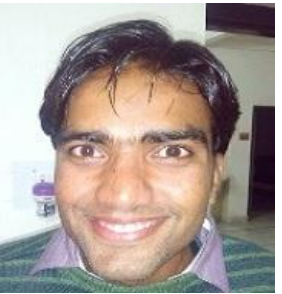

2

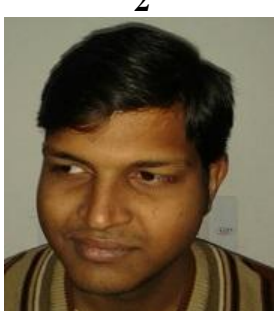

4

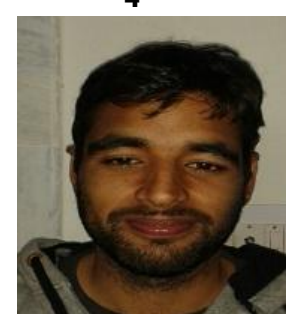

6

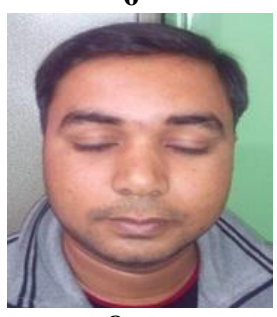

8

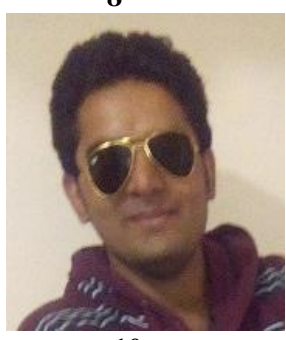

10
Fig.6: Train database

When the code is executed in MATLAB 2013a than its take the train database and test database path respectively according to figure 7

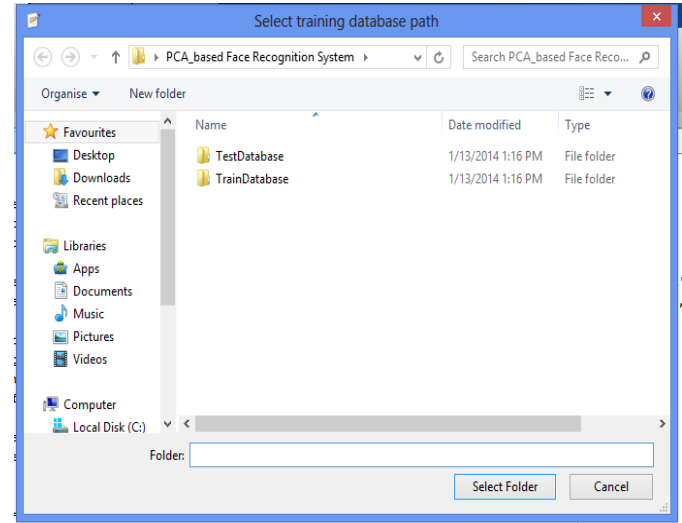

Fig. 7 select training database path

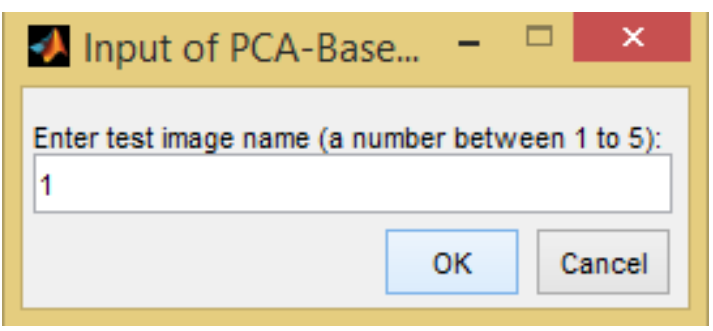

Fig.8: input which image to be matched
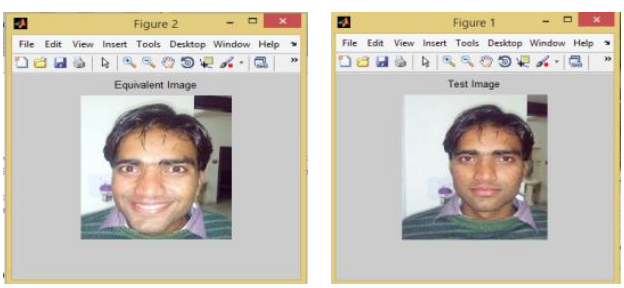

Fig 9: matched image

After successfully matching of image using PCA (principal component analysis) we can get much more accuracy level when we apply region base segmentation on both matched images and calculate gradient magnitude and match both gradient magnitude value if value is match approx. $60 \%$ than image is finally matched The experimental result is shown in figure 9 where two different pose of image is matched successfully the accuracy level is approx. $90 \%$ found

\section{CONCLUSION}

most of methods of making computers recognize faces were limited by the use of improvised face models and feature descriptions(matching simple distances), assuming that a face is no more than the sum of its parts, the individual features. This factual method using Principal Component Analysis for face recognition was actually actuate by information theory Face recognition on a small set of image features that best close set of known face images, without regarding that they correspond to our non-rational notions of facial parts and features. The Eigen face method and region base color segmentation matching provides a practical solution that is well fitted for the problem of face recognition. It is fast, relatively easy, and works well in a constrained environment. Head size, and head orientation, the tradeoffs between the number of Eigen faces necessary for unambiguous classification are matter of concern. 


\section{REFERENCES}

[1] Ming-Hsuan Yangwl David J. Kriegman and Narendra Ahuja, "Detecting Faces in Images", IEEE Transactions on Pattern Analysis and Machine Intelligence, vol 24, no. 1, pp. 696-706

[2] Kyungnam Kim, "Face Recognition using principal component analysis", USA, June 2000. Transaction Pattern Ana1ysis and Machine Intelligence 29 (1) (2007) $40-51$.

[3] D. Zhao, Z. Liu, R Xiao, X. Tang, Linear Laplacian discrimination for feature extraction, in: Proceedings of the IEEE Conference on Computer Vision and Pattern Recognition, 2007.

[4] Utah State University - Spring 2012 STAT 5570: Statistical Bioinformatics Notes 2.4

[5] "Elementary Linear Algebra 5e" by Howard Anton, Publisher John Wiley \& Sons Inc, ISBN 0-471-85223-6

[6] Issam Dagher and Rabih Nachar (2006). "Face Recognition Using IPCAICA Algorithm", IEEE transactions on Pattern Analysis and Machine Intelligence, vol. 28 ,

[7] Dr. H.B. Kekre et. al. / (IJCSE) International Journal on Computer Science and Engineering Vol. 02, No. 04, 2010, 959-964

[8] J. Yang, D. Zhang, and A. F. Frangi. Two-dimensional PCA: A new approach to appearance-based face representation and recognition. IEEE Transactions on Pattern Analysis and Machine Intelligence, 26(1):131137, 2004.

[9] A.A. Mohammed, R. Minhas, Q.M. Jonathan Wu, M.A. Sid-Ahmed Evaluation of face recognition technique using PCA, wavelets and SVM Pattern Recognition, Volume 44, Issues 10-11 elsevier2011

[10] Wankou Yang Laplacian bidirectional PCA for face recognition, Neuro computing, Volume 74,elsevier2010

[11] Karin Sobottka and Ioannis Pitas, "A Novel Method for Automatic Face Segmentation, Facial Feature Extraction and Tracking", Signal Processing: Image Communication, vol. 12, no.3, pp. 263-281, 1998. 